\title{
MWRRET Value-Added Product: The Retrieval of Liquid Water Path and Precipitable Water Vapor from Microwave Radiometer (MWR) Data Sets
}

Revision 2

KL Gaustad

DD Turner

SA McFarlane

July 2011

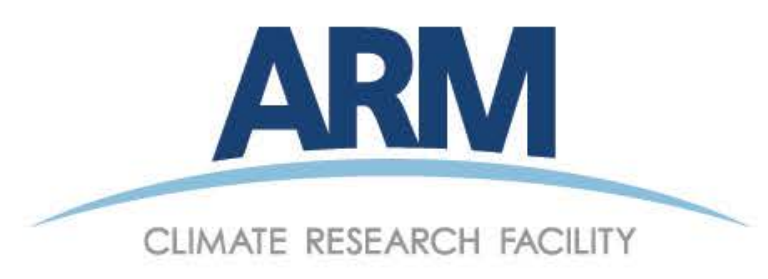




\section{DISCLAIMER}

This report was prepared as an account of work sponsored by the U.S. Government. Neither the United States nor any agency thereof, nor any of their employees, makes any warranty, express or implied, or assumes any legal liability or responsibility for the accuracy, completeness, or usefulness of any information, apparatus, product, or process disclosed, or represents that its use would not infringe privately owned rights. Reference herein to any specific commercial product, process, or service by trade name, trademark, manufacturer, or otherwise, does not necessarily constitute or imply its endorsement, recommendation, or favoring by the U.S. Government or any agency thereof. The views and opinions of authors expressed herein do not necessarily state or reflect those of the U.S. Government or any agency thereof. 


\title{
MWRRET Value-Added Product: The Retrieval of Liquid Water Path and Precipitable Water Vapor from Microwave Radiometer (MWR) Data Sets
}

\author{
KL Gaustad \\ DD Turner \\ SA McFarlane
}

July 2011

Work supported by the U.S. Department of Energy,

Office of Science, Office of Biological and Environmental Research 


\begin{abstract}
This report provides a short description of the Atmospheric Radiation Measurement (ARM) Climate Research Facility microwave radiometer (MWR) Retrieval (MWRRET) value-added product (VAP) algorithm. This algorithm utilizes a complementary physical retrieval method and applies brightness temperature offsets to reduce spurious liquid water path (LWP) bias in clear skies resulting in significantly improved precipitable water vapor (PWV) and LWP retrievals. We present a general overview of the technique, input parameters, output products, and describe data quality checks. A more complete discussion of the theory and results is given in Turner et al. (2007b).
\end{abstract}




\section{Contents}

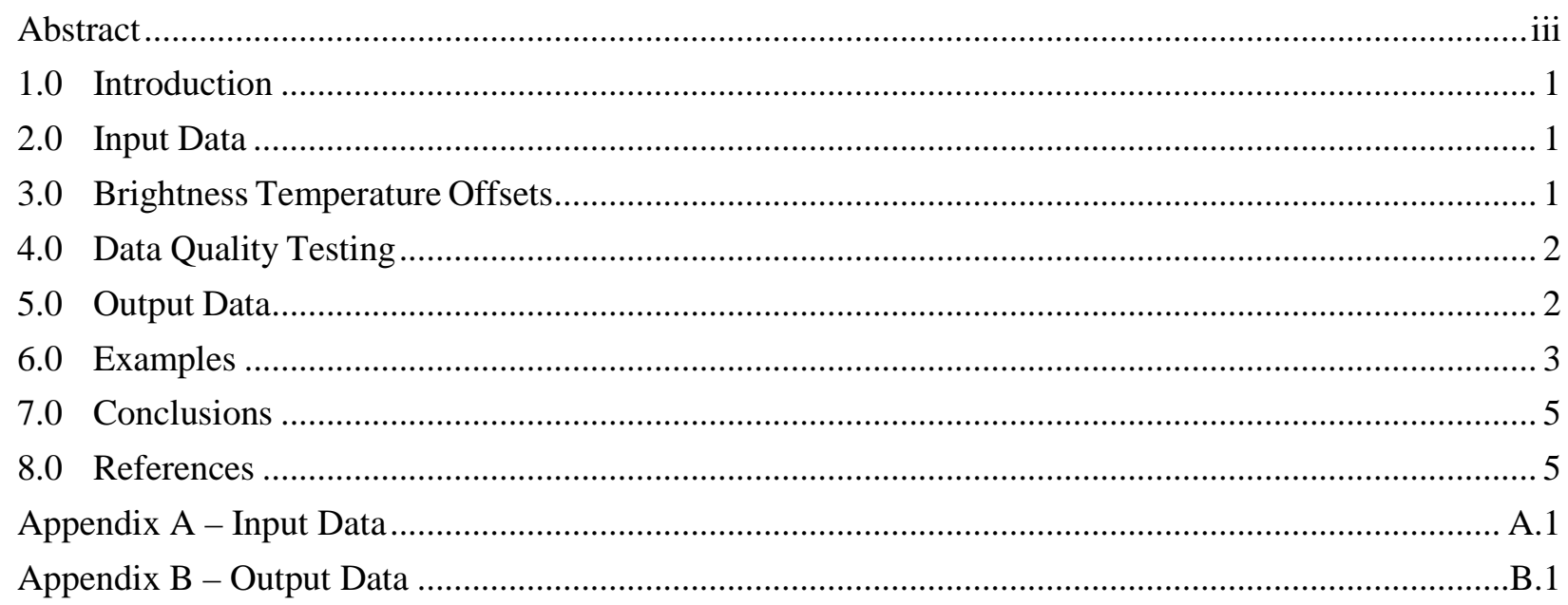




\subsection{Introduction}

The Atmospheric Radiation Measurement (ARM) Climate Research Facility deploys microwave radiometers (MWR) that observe downwelling radiation at 23.8 and $31.4 \mathrm{GHz}$ to each of its data gathering sites. Retrievals of precipitable water vapor (PWV) and liquid water path (LWP), crucial elements in building a better understanding of radiative transfer in the atmosphere and clouds, are generated from a retrieval algorithm that resides on the MWR instrument computers. These instrument-based retrieval algorithms use a statistical methodology based on site-dependent monthly retrieval coefficients (Liljegren and Lesht 1996). While very fast, this inversion approach can have large errors if atmospheric conditions are significantly different from the conditions captured in the retrieval coefficients, the retrieval coefficients are site-specific and cannot be applied to other locations, and the method does not address the systematic clear-sky biases in the retrieved LWP (Turner and Gaustad 2004). The MWR Retrieval (MWRRET) value-added product (VAP) was developed to address the inherent shortfalls in the original (orig) statistical retrieval algorithm that resides on the MWR. It does this through the use of a physical retrieval method and the application of brightness temperature offsets. The physical retrieval algorithm (phys) produces the most accurate retrievals possible when the atmospheric temperature profile and an estimate of the distribution of water vapor and liquid water are known.

The details of this algorithm have been published in Turner et al. (2007b). We refer the readers to this article for more detailed information about the algorithm applied in this VAP.

\subsection{Input Data}

The input data required by this VAP include observed brightness temperatures, PWV and LWP retrievals produced by the 'orig' algorithm residing on the MWR instrument, and surface meteorology observations. The actual datastream from which the surface meteorology data is extracted is a function of the site and facility that is being processed. Appendix A, Table A.1, lists the datastreams and correlating input and quality check fields that are required to run the MWRRET VAP. Optional inputs such as reflectivity, cloud base height, and vertical profiles of atmospheric conditions are presented in Appendix A, Table A.2. While the VAP will run without the balloon-borne sounding system profiles, they should be used when available. The specific sonde platform used as input is selected based on data availability. If more than one sonde platform is available for a given day, the input is chosen based on a prioritized list with 'wnpn.b1' the first choice, followed by 'wrpn.a1', 'wrpn.b1', 'wnpn.a1', and 'wrpr.a1'. Whether or not to use the cloud base height from the Belfort laser ceilometer (BLC), Vaisala ceilometer (VCEIL), or Active Remote Sensing of Clouds (ARSCL) VAP as input is specified at the command line and is left to the VAP operator's discretion.

\subsection{Brightness Temperature Offsets}

Offsets to brightness temperature can be applied to both the 23.8-GHz and 31.4-GHz channels. The 23.8GHz offsets, which reduce bias in the retrieved PWV, are determined once per year for each site and facility and as such are referred to as 'static' offsets. When running in real time on the ARM production system, static $T_{b}$ offsets are not applied. At the end of each year, the data from the year are post-analyzed 
and the appropriate static offset value at $23.8 \mathrm{GHz}$ is determined. The $\mathrm{T}_{\mathrm{b}}$ offset configuration file is updated with the value of newly determined static offsets, and the data are reprocessed to apply the offsets. For sites with consistently high precipitable water vapor (such as the Tropical Western Pacific sites of Manus and Nauru), the static offsets are not needed and are not applied. Additionally, static offsets are not calculated for sites (such as NSA C2 and SGP extended facilities) where radiosondes are not available.

As previously discussed, small biases in the retrieved LWP (from +/- 5 to $30 \mathrm{~g} / \mathrm{m} 2$ ) can be retrieved from the MWR in clear-sky scenes when the LWP should be zero (within the retrieval uncertainty). These LWP biases, which can exist for long periods of time, result in significant error in radiative transfer calculations (e.g., Turner et al. 2007a). To reduce the size of the LWP biases, the VAP also can be configured to subtract small offsets from the observed brightness temperature at $31.4 \mathrm{GHz}$ so that, statistically, a LWP of zero in clear skies is obtained. To capture the impact of calibration changes over time, these 'variable' offsets are computed directly from the data for a specified number of cases, which are kept in a rolling database of offsets stored in the $\mathrm{Tb}$ offset configuration file. The VAP computes the mean value from the middle two quartiles of the offset data to determine the value to subtract from the observation. This offset is added to the rolling database, and the oldest offset value is removed to maintain a fixed number of points in the rolling database. The variable offset is applied only to the 31.4-GHz channel. More details on the $\mathrm{Tb}$ offsets are given in Turner et al. 2007b.

\subsection{Data Quality Testing}

All quality flags associated with the input fields are propagated to the output. Additional quality tests, developed by Jim Liljegren, are applied to the MWR brightness temperatures to identify periods where these values experience sudden abnormal instantaneous changes in their values. These additional brightness temperature tests relate to instrument health as determined by examination of available data. Tests applied include identification of thermal stabilization issues, detection of spikes, and invalid optical depths. Quality control (QC) tests also were developed for the non-best-estimate PWV and LWP fields calculated by the VAP.

\subsection{Output Data}

The primary data products of the MWRRET VAP are best estimates of the PWV and LWP values. In addition to the best estimate values, the PWV and LWP values for the 'orig', 'stat2', and 'phys' methods are included in the output, as are supporting surface meteorological and MWR input data used in the physical retrieval calculations.

Given that yearly reprocessing at some sites is done to calculate static offsets and use the ASRCL data when available, two levels of output files, ${ }^{*} \mathrm{c} 1$ and ${ }^{*} \mathrm{c} 2$ can be produced by the VAP so that the user knows whether they have the final data set for the given site/time period. In general a ${ }^{*} 1$ level file is produced when it is expected that additional processing will occur for the given site/day and a *c2 level file is produced if no additional processing is expected (because static offsets have already been applied or do not exist for the site). For *c2 level files, global attributes read from the MWR input file are 
propagated to the output and new attributes are defined for the static Tb offset bias values (if they exist) indicating when these offsets were created. In addition, in the *c2 data level files be_pwv and be_lwp samples with indeterminate QC assessments have been set to the missing value of -9999. A complete list of all the output fields and a brief description of each is given in Appendix B.

One file is created for each day named with the following convention:

\section{XXXmwrret1liljclouFF.cN.YYYYMMDD.hhmmss}

where:

$$
\begin{aligned}
& \mathrm{XXX}=\text { the location of the instrument (nsa, sgp, twp, pye, etc.) } \\
& \text { mwrret1liljclou = identifies that this is Turner's version } 1 \text { MWRRET VAP } \\
& \text { FF = facility (e.g., C1) } \\
& \mathrm{N}=1 \text { or } 2 \\
& \text { YYYYMMDD = year, month, and day } \\
& \text { hhmmss = hour, minute, second }
\end{aligned}
$$

The VAP generates a quicklook output file within which three plots are presented: (1) MWR brightness temperature values including data quality status, (2) PWV calculated by the 'orig' and 'phys' retrieval methods, and (3) LWP calculated by the 'orig' and 'phys' retrieval methods. Two versions of this quicklook product are created. One version has a standard fixed y-axis for each of the plots, and another a dynamically resized y-axis to automatically adjust for periods with relatively flat brightness temperature and retrieval values. The output file for the standard and dynamically resized quicklooks uses the following naming convention:

\section{XXXmwrret1liljclouC1.YYYYMMDD.png \\ XXXmwrret1liljclouC1.YYYYMMDD.dynamic.png.}

\subsection{Examples}

Figure 1 shows the standard quicklook product for October 8, 1999. Using command line options, the MWRRET VAP allows the user to specify whether and how to perform the physical retrievals for a given day. Possible scenarios include running the physical retrieval for each sample in the day, for every nth sample, for 30 minutes after each sonde launch, or only for samples that correspond to a sonde launch. In production mode, the physical retrievals are performed on every sample in each day. The data quality test results are folded into the brightness temperature plot as colored bars along the bottom axis with the legend to the right of the plot. The legend only displays the tests that failed for that day. For October 8, 1999, the qc instrument health tests that failed include 'possible spike’ and 'spike simple.'

In Figure 2, the dynamically sized version of October 8, 1999, the blue line representing the physical retrieval sample value is evident just below the 'orig' retrieval values. 

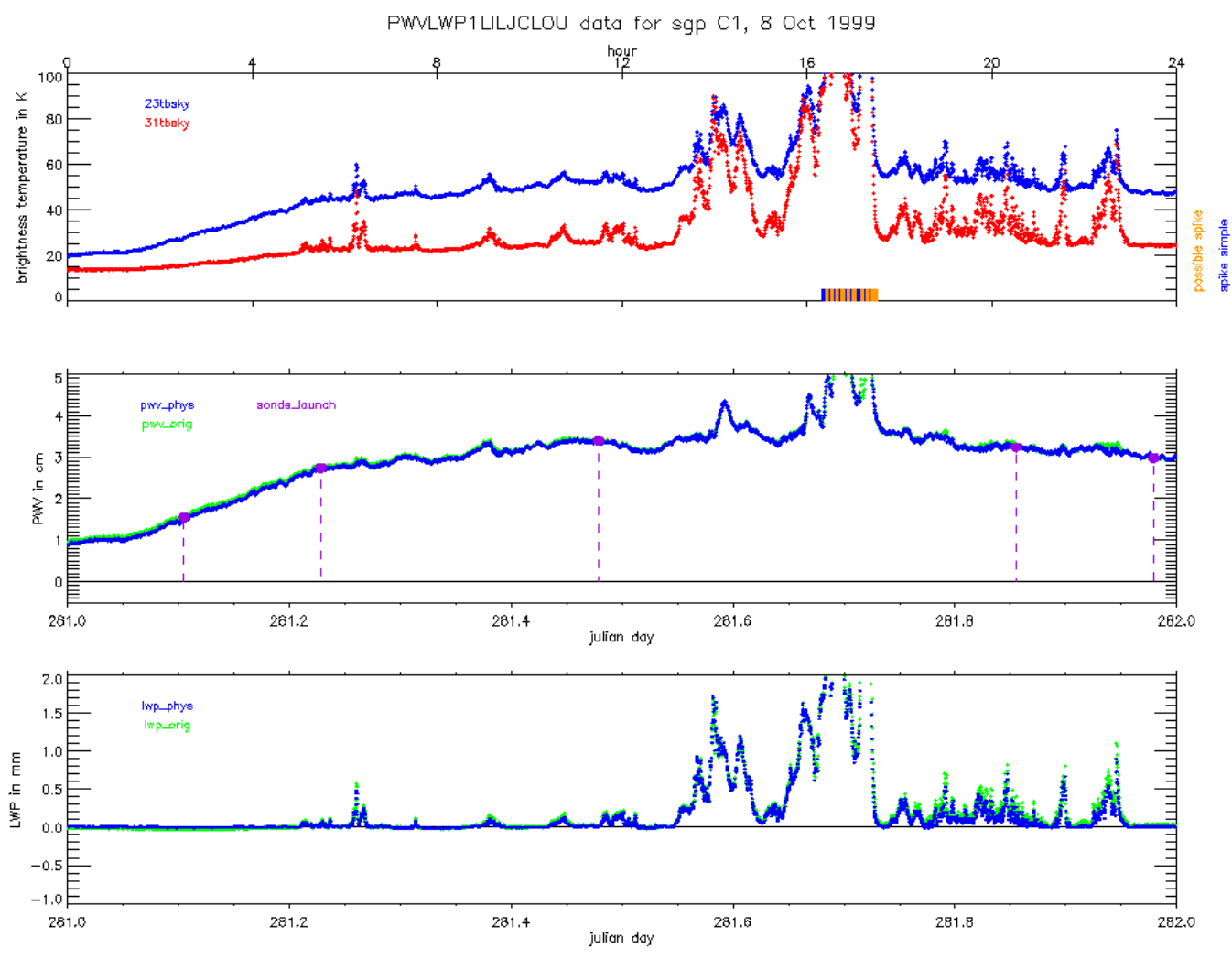

Figure 1. Quicklook image using standard y-axis ranges.
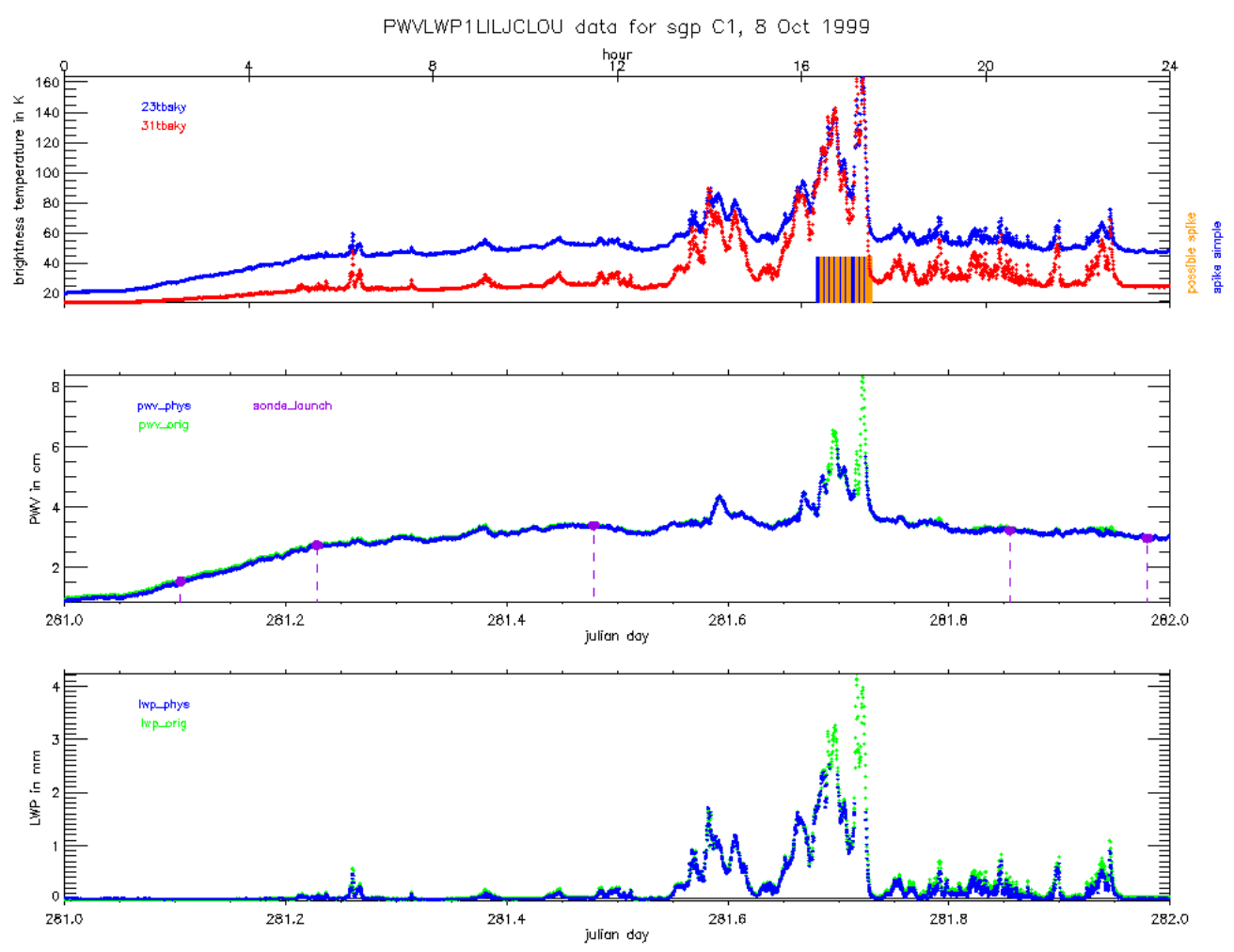

Figure 2. Quicklook image using dynamically sized y-axis. 


\subsection{Conclusions}

Measurements of both cloud properties and radiative fluxes are needed to improve global climate models (GCMs) and subsequently their prediction of future climates. Accurate measurements of PWV and LWP values support these goals through validating radiative transfer models, specifying atmospheric and cloud properties, and increasing the understanding of the first aerosol indirect effect. By providing an improved physical retrieval method and applying brightness temperature offsets to reduce spurious LWP bias in clear skies, the MWRRET algorithm provides significantly better retrievals of PWV and LWP from the ARM 2-channel microwave radiometers compared to the original retrieval method included in the ARM MWR ingest data product.

\subsection{References}

Liljegren, JC, and BM Lesht. 1996. "Measurements of integrated water vapor and cloud liquid water from microwave radiometers at the DOE ARM cloud and radiation testbed in the U.S. Southern Great Plains." In Proceedings of the International Geoscience and Remote Sensing Symposium (IGARSS), Lincoln, Nebraska, pp.1675-1677.

Turner, DD, and KL Gaustad. 2004. "Improved PWV and LWP retrievals from the microwave radiometer for ARM.” In Proceedings of the Fourteenth Atmospheric Radiation Measurement (ARM) Science Team Meeting, U.S. Department of Energy. Available URL: http://www.arm.gov/publications/proceedings/conf14/author.stm

Turner, DD, AM Vogelmann, R Austin, JC Barnard, K Cady-Pereira, C Chiu, SA Clough, CJ Flynn, MM Khaiyer, JC Liljegren, K Johnson, B Lin, CN Long, A Marshak, SY Matrosov, SA McFarlane, MA Miller, Q Min, P Minnis, W O’Hirok, Z Wang, and W Wiscombe. 2007a. “Optically thin liquid water clouds: Their importance and our challenge.” Bulletin of the American Meteorological Society 88: 191-204.

Turner, DD, SA Clough, JC Liljegren, EE Clouthiaux, K Cady-Pereira, and KL Gaustad. 2007b. "Retrieving liquid water path and precipitable water vapor from the Atmospheric Radiation Measurement (ARM) microwave radiometers.” IEEE Transactions on Geoscience and Remote Sensing 45(11): 36803689. 


\section{Appendix A Input Data}

Table A.1 lists the input datastreams and fields required to run the MWRRET VAP.

Table A.2 lists the input datastreams and fields that are optional inputs to the MWRRET VAP.

Table A.1. Required input files and variables.

\begin{tabular}{|c|c|c|c|}
\hline Datastream & Variable Name & Variable Long Name & Units \\
\hline \multirow{17}{*}{$\begin{array}{l}X X X m w r l o s F F . b 1 \\
X X X \text { is all sites }\end{array}$} & tbsky23 & 23.8 GHz sky brightness temperature & K \\
\hline & tbsky31 & $31.4 \mathrm{GHz}$ sky brightness temperature & $\mathrm{K}$ \\
\hline & vap & Total water vapor along LOS path & $\mathrm{cm}$ \\
\hline & liq & Total liquid water along LOS path & $\mathrm{cm}$ \\
\hline & sky23 & 23.8 GHz sky signal & counts \\
\hline & sky31 & 31.4 GHz sky signal & counts \\
\hline & teflon_window_correction_coef_23 & global attribute - no long name & unitless \\
\hline & teflon_window_correction_coef_31 & global attribute - no long name & unitless \\
\hline & mean_atmos_radiating_temp_23 & global attribute - no long name & unitless \\
\hline & mean_atmos_radiaing_temp_31 & global attribute - no long name & unitless \\
\hline & cosmic_background_temperature & global attribute - no long name & unitless \\
\hline & vapor_retrieval_coefficient_0 & global attribute - no long name & unitless \\
\hline & vapor_retrieval_coefficient_0 & global attribute - no long name & unitless \\
\hline & vapor_retrieval_coefficient_1 & global attribute - no long name & unitless \\
\hline & liquid_retrieval_coefficient_0 & global attribute - no long name & unitless \\
\hline & liquid_retrieval_coefficient_1 & global attribute - no long name & unitless \\
\hline & liquid_retrieval_coefficient_2 & global attribute - no long name & unitless \\
\hline \multirow{9}{*}{$\begin{array}{l}X X X \text { mwrlosFF.b1 } \\
X X X \text { is not a } \\
\text { parsl site }\end{array}$} & bb23 & 23.8 GHz Blackbody signal & counts \\
\hline & bb31 & 31.4 GHz Blackbody signal & counts \\
\hline & bbn23 & $\begin{array}{l}23.8 \mathrm{GHz} \text { blackbody+noise } \\
\text { injection signal }\end{array}$ & counts \\
\hline & bbn31 & $\begin{array}{l}\text { 31.4 GHz blackbody+noise } \\
\text { injection signal }\end{array}$ & counts \\
\hline & tkbb & Blackbody kinetic temperature & $\mathrm{K}$ \\
\hline & tkxc & Mixer kinetic (physical) temperature & $\mathrm{K}$ \\
\hline & tnd_nom23 & $\begin{array}{l}\text { Noise injection temp at } \\
\text { nominal temperature at } 23.8\end{array}$ & $\mathrm{~K}$ \\
\hline & tnd_nom31 & $\begin{array}{l}\text { Noise injection temp at } \\
\text { nominal temperature at } 31.4\end{array}$ & $\mathrm{~K}$ \\
\hline & tc23 & $\begin{array}{l}\text { Temperature correction coefficient at } \\
23.8 \mathrm{GHz}\end{array}$ & $\mathrm{K} / \mathrm{K}$ \\
\hline
\end{tabular}


Table A.1. Required input files and variables (cont.).

\begin{tabular}{|c|c|c|c|}
\hline Datastream & Variable Name & Variable Long Name & Units \\
\hline \multirow{5}{*}{$\begin{array}{l}X X X \text { mwrlosFF.b1 } \\
X X X \text { is not a } \\
\text { parsl site } \\
\text { (cont.) }\end{array}$} & tc31 & $\begin{array}{l}\text { Temperature correction coefficient at } \\
31.4 \mathrm{GHz}\end{array}$ & $\mathrm{K} / \mathrm{K}$ \\
\hline & qc_tbsky23 & $\begin{array}{l}\text { Quality check results on field: } 23.8 \\
\text { GHz sky brightness temperature }\end{array}$ & unitless \\
\hline & qc_tbsky31 & $\begin{array}{l}\text { Quality check results on field: } 31.4 \\
\text { GHz sky brightness temperature }\end{array}$ & unitless \\
\hline & qc_vap & $\begin{array}{l}\text { Quality check results on field: } \\
\text { Total water vapor along LOS }\end{array}$ & unitless \\
\hline & qc_liq & $\begin{array}{l}\text { Quality check results on field: } \\
\text { Total liquid water along LOS }\end{array}$ & unitless \\
\hline \multirow{4}{*}{$\begin{array}{l}X X X 1 \text { twrmwrFF.c1 } \\
X X X \text { is sgp } \\
\text { FF is C1 or E13 }\end{array}$} & pres_02 & Barometric pressure at $2 \mathrm{~m}$ & $\mathrm{mb}$ \\
\hline & temp_02 & Temperature at $2 \mathrm{~m}$ & $\operatorname{deg} C$ \\
\hline & vap_pres_02m & Vapor pressure at $2 \mathrm{~m}$ & $\mathrm{mb}$ \\
\hline & rh_02 & Relative humidity at $2 \mathrm{~m}$ & $\%$ \\
\hline \multirow{8}{*}{$\begin{array}{l}\text { XXXthwapsFF.b1 } \\
\text { where XXX is sgp } \\
\text { FF is not C1 or } \\
\text { E13 }\end{array}$} & pres & Pressure & $\mathrm{hPa}$ \\
\hline & temp & Temperature & C \\
\hline & vap_res & Vapor pressure & $\mathrm{kPa}$ \\
\hline & rh & Relative humidity & $\%$ \\
\hline & qc_press & Quality check results on field: & unitless \\
\hline & qc_temp & $\begin{array}{l}\text { Quality check results on } \\
\text { field: Temperature }\end{array}$ & unitless \\
\hline & qc_vap_pres & $\begin{array}{l}\text { Quality check results on field: Vapor } \\
\text { pressure }\end{array}$ & unitless \\
\hline & qc_rh & $\begin{array}{l}\text { Quality check results on field: Vapor } \\
\text { pressure }\end{array}$ & unitless \\
\hline \multirow{7}{*}{$\begin{array}{l}\text { XXXsmet60sFF.b1 } \\
X X X \text { is twp or } \\
\text { pye and not a } \\
\text { parsl site }\end{array}$} & atmos_pressure & Pressure & \\
\hline & temp_mean & Temperature & \\
\hline & vappress_mean & Vapor pressure & \\
\hline & relh_mean & Relative humidity & \\
\hline & qc_atmos_pressure & Quality check results on field: & unitless \\
\hline & qc_temp_mean & $\begin{array}{l}\text { Quality check results on } \\
\text { field: Temperature }\end{array}$ & unitless \\
\hline & qc_relh_mean & $\begin{array}{l}\text { Quality check results on field: Vapor } \\
\text { pressure }\end{array}$ & unitless \\
\hline \multirow{2}{*}{$\begin{array}{l}X X X \text { mettwr4hFF.b1 } \\
X X X \text { is nsa }\end{array}$} & AtmPress & Atmospheric pressure & $\mathrm{kPa}$ \\
\hline & T2M_AVG & $2 \mathrm{~m}$ average temperature & C \\
\hline
\end{tabular}


Table A.1. Required input files and variables (cont.).

\begin{tabular}{|c|c|c|c|}
\hline Datastream & Variable Name & Variable Long Name & Units \\
\hline \multirow[t]{6}{*}{ FF is C1 } & VP2M_AVG & $2 m$ average calculated vapor & $\mathrm{kPa}$ \\
\hline & RH2M_AVG & $2 m$ average relative humidity & $\%$ \\
\hline & qc_AtmPress & $\begin{array}{l}\text { Quality check results on } \\
\text { field: Atmospheric pressure }\end{array}$ & unitless \\
\hline & qc_T2M_AVG & $\begin{array}{l}\text { Quality check results on field: } 2 \mathrm{~m} \\
\text { average temperature }\end{array}$ & unitless \\
\hline & qc_VP2M_AVG & $\begin{array}{l}\text { Quality check results on field: } 2 \mathrm{~m} \\
\text { average calculated vapor pressure }\end{array}$ & unitless \\
\hline & qc_RH2M_AVG & $\begin{array}{l}\text { Quality check results on field: } 2 \mathrm{~m} \\
\text { average relative humidity }\end{array}$ & unitless \\
\hline \multirow{8}{*}{$\begin{array}{l}X X X \text { mettwr2hFF.b1 } \\
X X X \text { is nsa } \\
F F \text { is C2 }\end{array}$} & AtmPress & Atmospheric pressure & $\mathrm{kPa}$ \\
\hline & T2m_AVG & $2 \mathrm{~m}$ average temperature & C \\
\hline & VP2m_AVG & $2 \mathrm{~m}$ average calculated vapor & $\mathrm{kPa}$ \\
\hline & RH2m_AVG & $2 m$ average relative humidity & $\%$ \\
\hline & qc_AtmPress & $\begin{array}{l}\text { Quality check results on } \\
\text { field: Atmospheric pressure }\end{array}$ & unitless \\
\hline & qc_T2m_AVG & $\begin{array}{l}\text { Quality check results on field: } 2 \mathrm{~m} \\
\text { average temperature }\end{array}$ & unitless \\
\hline & qc_PV2m_AVG & $\begin{array}{l}\text { Quality check results on field: } 2 \mathrm{~m} \\
\text { average calculated vapor pressure }\end{array}$ & unitless \\
\hline & qc_RH2m_AVG & $\begin{array}{l}\text { Quality check results on field: } 2 \mathrm{~m} \\
\text { average relative humidity }\end{array}$ & unitless \\
\hline \multirow{3}{*}{$\begin{array}{l}X X X \text { mettwrFF.b1 } \\
X X X \text { is a parsl site }\end{array}$} & pressure & Surface atmospheric pressure & $\begin{array}{l}\text { hecto- } \\
\text { Pascals } \\
\text { (milibars) }\end{array}$ \\
\hline & temperature & $\begin{array}{l}\text { Surface atmospheric dry } \\
\text { bulb temperature }\end{array}$ & $\begin{array}{l}\text { degrees } \\
\text { Celsius }\end{array}$ \\
\hline & rh & Surface atmospheric relative humidity & Percent \\
\hline
\end{tabular}


Table A.2. Optional input files and variables.

\begin{tabular}{|c|c|c|c|}
\hline Datastream & Variable Name & Variable Long Name & Units \\
\hline \multirow{4}{*}{ XXXsonde<type>FF.b1 } & alt & Altitude & $\begin{array}{l}\text { meters } \\
\text { above } \\
\text { Mean } \\
\text { Sea }\end{array}$ \\
\hline & pres & Pressure & $\mathrm{hPa}$ \\
\hline & tdry & Dry Bulb Temperature & C \\
\hline & rh & Relative Humidity & $\%$ \\
\hline \multirow{3}{*}{ XXXarscl1clothFF.c1 } & CloudBaseBestEstimate & $\begin{array}{l}\text { Cloud base height best } \\
\text { estimate }\end{array}$ & m AGL \\
\hline & Heights & Height of measured value & m AGL \\
\hline & ReflectivityBestEstimate & $\begin{array}{l}\text { MCR best estimate of } \\
\text { hydrometeor reflectivity }\end{array}$ & $\begin{array}{l}\mathrm{dBZ} \\
(X 100)\end{array}$ \\
\hline \multirow{2}{*}{$\begin{array}{l}\text { XXXvceil25kFF.a1 } \\
\text { XXXvceil25kFF.b1 }\end{array}$} & first_cbh & $\begin{array}{l}\text { Lowest cloud base } \\
\text { height detected }\end{array}$ & $\mathrm{m}$ \\
\hline & detection_status & Detection status & unitless \\
\hline XXXblcFF.a1 & cloud1 & $\begin{array}{l}\text { Base height of lowest cloud } \\
\text { detected by threshold }\end{array}$ & $\begin{array}{l}\mathrm{m} \\
\text { above } \\
\text { ground }\end{array}$ \\
\hline
\end{tabular}

The names of the input datastreams have the format:

XXX $<$ plat $>$ FF. $<$ level $>$.YYYYMMDD.hhm

mss

Where:

$\mathrm{XXX}=$ the locations of the instrument (nsa, sgp, twp, pye, etc.)

$<$ plat $>=$ the main instrument name

$\mathrm{FF}=$ facility (e.g., C1)

$<$ level $>$ = data level, can be a1

YYYYMMDD = year, month, and day hhmmss = hour, minute, second 


\section{Appendix B Output Data}

Table B.1 lists the fields found in the MWRRET output netCDF files.

Table B.1. Output variables.

\begin{tabular}{|c|c|c|}
\hline Fieldname & Description & Units \\
\hline base_time & Base time in epoch & $\begin{array}{l}\text { seconds since } \\
\text { 1970/01/01 } \\
00: 00: 00\end{array}$ \\
\hline time_offset & Time offset from base_time & $\begin{array}{l}\text { seconds since } \\
\text { base_time }\end{array}$ \\
\hline time & Time offset from midnight & $\begin{array}{l}\text { seconds since } \\
\text { midnight }\end{array}$ \\
\hline hour & Hour of the day & UTC \\
\hline level_height & Level height & km AGL \\
\hline be_pwv & Precipitable water vapor best-estimate value & $\mathrm{cm}$ \\
\hline qc_be_pwv & $\begin{array}{l}\text { Quality check results on field: Precipitable water } \\
\text { vapor best-estimate value }\end{array}$ & unitless \\
\hline be_Iwp & Liquid water path best-estimate value & $\mathrm{g} / \mathrm{m}^{\wedge} 2$ \\
\hline qc_be_Iwp & $\begin{array}{l}\text { Quality check results on field: Liquid water path } \\
\text { best-estimate value }\end{array}$ & unitless \\
\hline cloud_base_height & Cloud base height & km AGL \\
\hline qc_cloud_base_height & Quality check results on field: Cloud base height & unitless \\
\hline cloud_temp & Cloud temperature used in the retrieval & $\mathrm{K}$ \\
\hline qc_cloud_temp & $\begin{array}{l}\text { Quality check results on field: Cloud temperature } \\
\text { used in the retrieval }\end{array}$ & unitless \\
\hline surface_temp & Surface temperature & $\mathrm{K}$ \\
\hline qc_surface_temp & Quality check results on field: Surface & unitless \\
\hline surface_vapor_pres & Surface vapor pressure & $\mathrm{kPa}$ \\
\hline qc_surface_vapor_pres & $\begin{array}{l}\text { Quality check results on field: Surface vapor } \\
\text { pressure }\end{array}$ & unitless \\
\hline surface_pres & Surface pressure & $\mathrm{kPa}$ \\
\hline qc_surface_pres & Quality check results on field: Surface pressure & unitless \\
\hline surface_rh & Surface relative humidity & $\%$ \\
\hline qc_surface_rh & $\begin{array}{l}\text { Quality check results on field: Surface relative } \\
\text { humidity" }\end{array}$ & unitless \\
\hline tbsky23 & Sky brightness temperature at $23.8 \mathrm{GHz}$ & $\mathrm{K}$ \\
\hline qc_tbsky23 & $\begin{array}{l}\text { Quality check results on field: Sky brightness } \\
\text { temperature at } 23.8 \mathrm{GHz}\end{array}$ & unitless \\
\hline tbsky31 & Sky brightness temperature at $31.4 \mathrm{GHz}$ & $\mathrm{K}$ \\
\hline qc_tbsky31 & $\begin{array}{l}\text { Quality check results on field: Sky brightness } \\
\text { temperature at } 31.4 \mathrm{GHz}\end{array}$ & unitless \\
\hline orig pwv & Total water vapor along LOS path & $\mathrm{cm}$ \\
\hline
\end{tabular}


Table B.1. Output variables (cont.).

\begin{tabular}{|c|c|c|}
\hline Fieldname & Description & Units \\
\hline qc_orig_pwv & $\begin{array}{l}\text { Quality check results on field: Total water vapor } \\
\text { along LOS path }\end{array}$ & unitless \\
\hline orig_Iwp & Total liquid water along LOS path & $\mathrm{g} / \mathrm{m}^{\wedge} 2$ \\
\hline qc_orig_Iwp & $\begin{array}{l}\text { Quality check results on field: Total liquid water } \\
\text { along LOS path" }\end{array}$ & unitless \\
\hline tbsky23_nobias & $\begin{array}{l}\text { Sky brightness temperature at } 23.8 \mathrm{GHz} \text {, with the } \\
\text { bias removed }\end{array}$ & K \\
\hline tbsky31_nobias & $\begin{array}{l}\text { Sky brightness temperature at } 31.4 \mathrm{GHz} \text {, with the } \\
\text { bias removed }\end{array}$ & K \\
\hline tbsky23 calculated & Calculated sky brightness temperature at $23.8 \mathrm{GHz}$ & $\mathrm{K}$ \\
\hline qc_tbsky23_calculated & $\begin{array}{l}\text { Quality check results on field: Calculated sky } \\
\text { brightness temperature at } 23.8 \mathrm{GHz}\end{array}$ & unitless \\
\hline tbsky31_calculated & Calculated sky brightness temperature at $31.4 \mathrm{GHz}$ & $\mathrm{K}$ \\
\hline qc_tbsky31_calculated & $\begin{array}{l}\text { Quality check results on field: Calculated sky } \\
\text { brightness temperature at } 31.4 \mathrm{GHz}\end{array}$ & unitless \\
\hline phys_pwv & $\begin{array}{l}\text { Precipitable water vapor retrieved using a } \\
\text { physical/iterative approach }\end{array}$ & $\mathrm{cm}$ \\
\hline qc_phys_lwp & $\begin{array}{l}\text { Quality check results on field: Precipitable water } \\
\text { vapor retrieved using a physical/iterative approach }\end{array}$ & unitless \\
\hline phys_Iwp & $\begin{array}{l}\text { Cloud liquid water path retrieved using a } \\
\text { physical/iterative approach }\end{array}$ & $\mathrm{g} / \mathrm{m}^{\wedge} 2$ \\
\hline qc_phys_lwp & $\begin{array}{l}\text { Quality check results on field: Cloud liquid water } \\
\text { path retrieved using a physical/iterative approach }\end{array}$ & unitless \\
\hline phys_pwv_uncertainty & $\begin{array}{l}\text { 1-sigma uncertainty in precipitable water vapor } \\
\text { retrieved using a physical/iterative approach }\end{array}$ & $\mathrm{cm}$ \\
\hline qc_phys_pwv_uncertainty & $\begin{array}{l}\text { Quality check results on field: 1-sigma uncertainty } \\
\text { in precipitable water vapor retrieved using } \\
\text { a physical/iterative approach }\end{array}$ & unitless \\
\hline phys_Iwp_uncertainty & $\begin{array}{l}\text { 1-sigma uncertainty in cloud liquid water path } \\
\text { retrieved using a physical/iterative approach }\end{array}$ & $\mathrm{g} / \mathrm{m}^{\wedge} 2$ \\
\hline qc_phys_Iwp_uncertainty & $\begin{array}{l}\text { Quality check results on field: 1-sigma uncertainty } \\
\text { in cloud liquid water path retrieved using } \\
\text { a physical/iterative approach }\end{array}$ & unitless \\
\hline phys_niter & $\begin{array}{l}\text { Number of iterations needed by the physical } \\
\text { retrieval }\end{array}$ & counts \\
\hline qc_phys_niter & $\begin{array}{l}\text { Quality check results on field: Number of iterations } \\
\text { needed by the physical retrieval for convergence }\end{array}$ & unitless \\
\hline phys_rms & $\begin{array}{l}\text { Root mean square difference between the } \\
\text { computed } \\
\text { and observed brightness temperatures for the }\end{array}$ & K \\
\hline
\end{tabular}


Table B.1. Output variables (cont.).

\begin{tabular}{|c|c|c|}
\hline Fieldname & Description & Units \\
\hline qc_phys_rms & $\begin{array}{l}\text { Quality check results on field: Root mean square } \\
\text { difference between the computed and } \\
\text { observed brightness temperatures for the }\end{array}$ & unitless \\
\hline phys_converge & Convergence value for the physical retrieval & arbitrary \\
\hline qc_phys_converge & $\begin{array}{l}\text { Quality check results on field: Convergence } \\
\text { value }\end{array}$ & unitless \\
\hline sonde_times & Flag indicating when the sonde launches & unitless \\
\hline sonde_pwv & $\begin{array}{l}\text { Precipitable water vapor integrated from the } \\
\text { radiosonde profile }\end{array}$ & $\mathrm{cm}$ \\
\hline qc_sonde_pwv & $\begin{array}{l}\text { Quality check results on field: Precipitable water } \\
\text { vapor integrated from the radiosonde profile }\end{array}$ & unitless \\
\hline mean_pwv_mwr & $\begin{array}{l}\text { Ensemble average for MWR vapor in window } \\
\text { centered upon current sample }\end{array}$ & $\mathrm{cm}$ \\
\hline sdev_pwv_mwr & $\begin{array}{l}\text { Standard deviation of ensemble average for } \\
\text { MWR }\end{array}$ & $\mathrm{cm}$ \\
\hline num_pwv_mwr & Size of MWR ensemble for mean_pwv_mwr field & unitless \\
\hline mean_lwp_mwr & $\begin{array}{l}\text { Ensemble average for MWR liquid in window } \\
\text { centered upon current sample }\end{array}$ & $\mathrm{g} / \mathrm{m}^{\wedge} 2$ \\
\hline sdev_Iwp_mwr & $\begin{array}{l}\text { Standard deviation of ensemble average for } \\
\text { MWR }\end{array}$ & $\mathrm{g} / \mathrm{m}^{\wedge} 2$ \\
\hline num_Iwp_mwr & Size of MWR ensemble for mean_Iwp_mwr field & unitless \\
\hline mean_tbsky23_mwr & $\begin{array}{l}\text { Ensemble average for MWR } 23.8 \mathrm{GHz} \text { sky } \\
\text { brightness temperature in window centered } \\
\text { upon current sample }\end{array}$ & $\mathrm{K}$ \\
\hline qc_mean_tbsky23_mwr & $\begin{array}{l}\text { Quality check results on field: Ensemble average } \\
\text { for } \\
\text { MWR } 23.8 \mathrm{GHz} \text { sky brightness }\end{array}$ & unitless \\
\hline sdev_tbsky23_mwr & $\begin{array}{l}\text { Standard deviation for ensemble average for } \\
\text { MWR }\end{array}$ & $\mathrm{K}$ \\
\hline qc_sdev_tbsky23_mwr & $\begin{array}{l}\text { Quality check results on field: Standard deviation } \\
\text { for ensemble average for MWR } 23.8 \mathrm{GHz} \\
\text { sky brightness temperature }\end{array}$ & unitless \\
\hline num_tbsky23_mwr & $\begin{array}{l}\text { Size of MWR ensemble for mean_tbsky23_mwr } \\
\text { field }\end{array}$ & unitless \\
\hline mean_tbsky31_mwr & $\begin{array}{l}\text { Ensemble average for MWR } 31.4 \mathrm{GHz} \text { sky } \\
\text { brightness temperature in window centered } \\
\text { upon current sample }\end{array}$ & $\mathrm{K}$ \\
\hline
\end{tabular}


Table B.1. Output variables (cont.).

\begin{tabular}{|l|l|l|}
\hline qc_mean_tbsky31_mwr & $\begin{array}{l}\text { Quality check results on field: Ensemble average } \\
\text { for } \\
\text { MWR } 31.4 \mathrm{GHz} \text { sky brightness }\end{array}$ & unitless \\
\hline sdev_tbsky31_mwr & $\begin{array}{l}\text { Standard deviation for ensemble average for } \\
\text { MWR }\end{array}$ & $\mathrm{K}$ \\
\hline qc_sdev_tbsky31_mwr & $\begin{array}{l}\text { Quality check results on field: Standard deviation } \\
\text { for ensemble average for MWR 31.4 GHz } \\
\text { sky brightness temperature }\end{array}$ & unitless \\
\hline num_tbsky31_mwr & $\begin{array}{l}\text { Size of MWR ensemble for mean_tbsky23_mwr } \\
\text { field }\end{array}$ & unitless \\
\hline vbias_yyyymmdd & Variable bias date & $\begin{array}{l}\text { YYYYMMDD } \\
\text { [UTC] }\end{array}$ \\
\hline vbias_hour & Variable bias hour & hour [UTC] \\
\hline vbias_23 & Variable bias offset at 23.8 GHz & K \\
\hline vbias_31 & Variable bias offset at 31.4 GHz & K \\
\hline lat & north latitude & degrees \\
\hline lon & east longitude & degrees \\
\hline alt & altitude & altitude \\
\hline
\end{tabular}




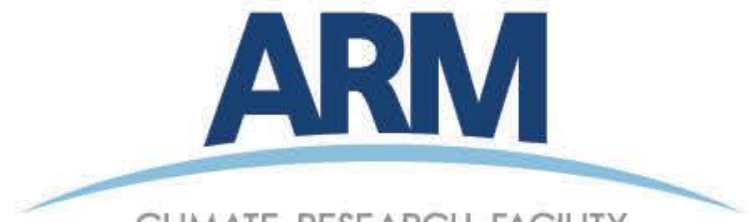

CLIMATE RESEARCH FACILITY

www.arm.gov

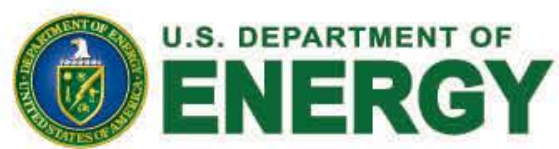

Office of Science 